\title{
Calcifediol Anhydrous
}

National Cancer Institute

\section{Source}

National Cancer Institute. Calcifediol Anhydrous. NCI Thesaurus. Code C77155.

The anhydrous form of calcifediol, a hormone precursor that is produced by the hydroxylation of vitamin $D$ in the liver. Calcifediol can be further hydroxylated in the kidney to give calcitriol, the active form of vitamin D. Plasma levels of this compound are measured to determine the vitamin D status of patients. 